Case Report:

\title{
Spinal Subdural Hematoma With Paraplegia Mimicking Dorsolumbar Prolapsed Disc: A Case Report
}

\author{
Vishal Singh $^{1^{*}}$, Sajad Hussain Arif ${ }^{1} \mathrm{O}$
}

1. Department of Neurosurgery, Sher-i-Kashmir Institute of Medical Sciences, Srinagar, India

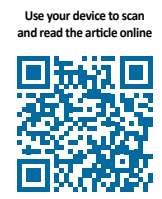

citation: Singh V, Arif SH. Spinal Subdural Hematoma With Paraplegia Mimicking Dorsolumbar Prolapsed Disc: A Case Report. Iran J Neurosurg. 2021; 7(2):113-118. http://dx.doi.org/10.32598/irjns.7.2.6

: http://dx.doi.org/10.32598/irjns.7.2.6

Article info:

Received: 26 Feb 2021

Accepted: 10 Mar 2021

Available Online: 01 Apr 2021

Keywords:

Subdural hematoma, Paraplegia, Prolapsed disc, Lumbar puncture, Conus medullaris

\section{A B STRACT}

Background and Importance: Spinal Subdural Hematoma (SSH) is a rare condition with an unknown incidence in the general population. latrogenic spinal subdural hematoma radiologically mimicking a prolapsed dorsolumbar disc has not been published in the literature.

Case Presentation: A 65-year-old female presented with altered sensorium and generalized weakness for 3 days evaluated by a neurologist who diagnosed it as a metabolic encephalopathy with Syndrome of Inappropriate Antidiuretic Hormone secretion (SIADH) with severe anemia and hypothyroidism. She underwent a diagnostic lumbar puncture and following which, within 24 hours, she developed weakness of both the lower limbs. MRI of dorsolumbar spine was done which revealed D12-L1 extruded disc causing severe compression to the conus medullaris with D12-L2 subarachnoid lesion and cord edema. On opening the dura, a large organized hematoma on the anterior aspect of the conus was seen and evacuated.

Conclusion: Spinal subdural hematoma presents with a spectrum of signs and symptoms. Although MRI is the investigation of choice but SSH presenting like a prolapsed intervertebral disc on MR is one of its kinds and should be kept in mind since it carries a grave prognosis if not treated early with emergency decompression.

\section{* Corresponding Author:}

Vishal Singh, MD.

Address: Department of Neurosurgery, Sher-i-Kashmir Institute of Medical Sciences, Srinagar, India

Tel: +91 (97) 84686370

E-mail:dr.vishalsingh1689@gmail.com 


\section{Highlights}

- latrogenic spinal subdural hematoma radiologically mimicking a prolapsed dorsolumbar disc has not been published in the literature.

- A 65-year-old female was posted for D12-L1 discectomy and was found to have SSH intra-operatively which mimicked disc prolapse on MRI.

- Although MRI is the investigation of choice, SSH presenting, like a prolapsed intervertebral disc on MRI is among its kinds and should be considered because it carries a grave prognosis if not treated early with emergency decompression.

\section{Plain Language Summary}

Spinal subdural hematoma (SSH) is a rare condition in the general population and radiologically similar to a disc prolapse that has not been published in the literature. We are presenting a 65-year-old female referred to us with inappropriate talks and generalized weakness evaluated by the Neurology department for 3 days and diagnosed as a cerebral medical condition with severe anemia and low thyroid hormone. She underwent a diagnostic CSF tap from the lower spine (lumbar puncture), following which, within 24 hours, she developed weakness of both the lower limbs. An MRI was performed, which revealed D12-L1 spinal disc prolapse causing severe compression to the spinal cord. The patient was taken up for surgery, and on opening the dura, a sizeable organized blood clot in front of the lower end of the spinal cord was seen and carefully removed. The patient remained paraplegic on the follow-up, which indicates that spinal subdural hematoma carries a lousy outcome and needs an early diagnostic modality like MRI. Also, on MRI, there is a possibility that a subdural hematoma can show the features of the prolapsed disc or disc bulge, which can mislead the surgeons for a delayed treatment and should be kept in mind.

\section{Background and Importance}

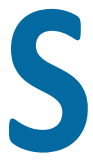

pinal Subdural Haematoma (SSH) is a rare condition with an unknown incidence in the general population. The iatrogenic SSH following diagnostic lumbar puncture has been linked to coagulopathies, vascular malformations, and bleeding disorders [1]. Usually, any insult to the vascular structure during Lumbar Puncture (LP) would lead to hematoma; accordingly, this condition may cause mid-back pain or skin discoloration, ultimately resolving without major complications. However, rarely, if the collection is near or inside the dural sac, it exerts a mass effect on the nerve root or the cord; subsequently, it can lead to myelopathy or Cauda Equina Syndrome (CES), requiring urgent decompression to prevent irreversible paralysis or death [2]. Given the rare condition and atypical presentation by patients, there is often delay in the diagnosis, leading to a poor prognosis of the condition [3]. The mortality rate of SSH equals $1.3 \%$ with the relevant morbidity being $28 \%$ [4].

latrogenic SSH radiologically mimicking a prolapsed dorsolumbar disc remains unaddressed in the literature. We presented the first case of SSH with paraplegia, following a diagnostic lumbar puncture which mimicked a D12-L1 disc extrusion with cauda equina syndrome on MRI.

\section{Case Presentation}

A 65-year-old female presented with altered sensorium and generalized weakness for 3 days. The patient was evaluated by a neurologist and diagnosed with metabolic encephalopathy with SIADH with severe anemia and hypothyroidism. For further evaluation, she underwent a diagnostic lumbar puncture at the level of L3-L4. Consequently, within 24 hours, she developed weakness of both lower limbs with the loss of bowel and bladder control along with loss of sensation below the pubic region. The reported patient was not on anticoagulant therapy. On examination, the studied patient manifested complete paraplegia, hypoesthesia below T12 dermatome, and absent lower limb reflexes with the loss of anal tone.

A Magnetic Resonance Imaging (MRI) of the dorsolumbar spine was conducted. The MRI data revealed D12-L1 extruded disc causing severe compression to the conus medullaris with D12-L2 subarachnoid lesion and cord edema (Figures 1 and 2). X-ray and Computerized Tomography (CT) scan of the spine results were normal. On further investigation, she presented $7.1 \mathrm{~g} / \mathrm{dl}$ hemoglobin, normal platelet count, prothrombin time of 13.8 with International Normalized Ratio (INR) and Partial Thromboplastin Time (PTT) of 1.11 and 24.4 , respectively. 


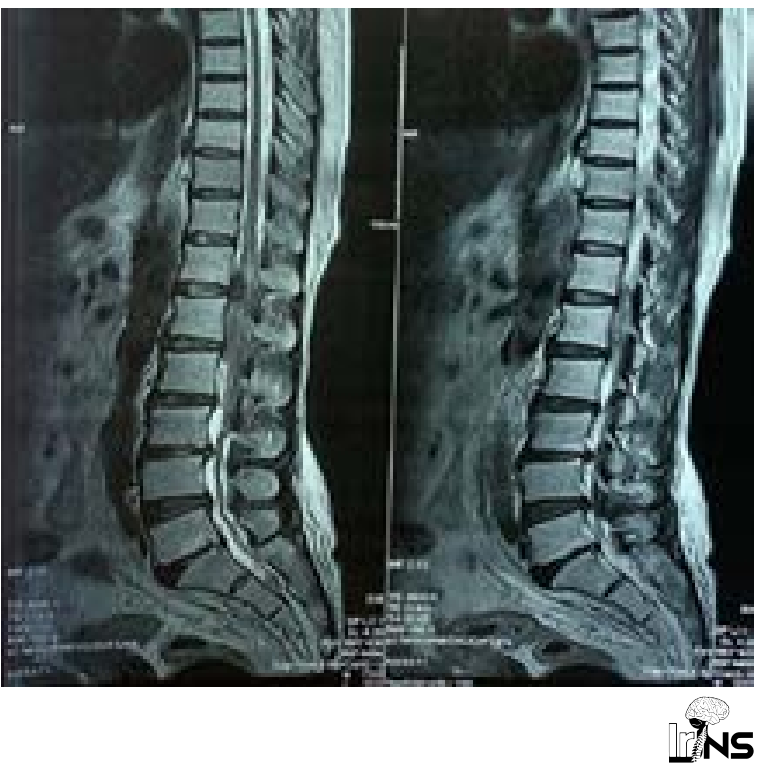

Figure 1. Sagittal T2WI of MRI of lumbosacral spine suggesting D12-L1 disc prolapse compressing the cord with distal cord edema

Moreover, $600 \mathrm{~mL}$ of whole blood was transfused and the reported patient was prepared for surgery. D12 laminectomy was performed to assess the D12-L1 disc; however, there was no disc extrusion or bulge at that level. Laminectomy was extended from complete $\mathrm{L} 1$ to partial D11. On opening the dura, a large organized hematoma on the anterior aspect of the conus was detected and evacuated (Figures 3 and 4). No obvious vascular malformations were found during surgery. The tissue retrieved was provided for histopathological examina-

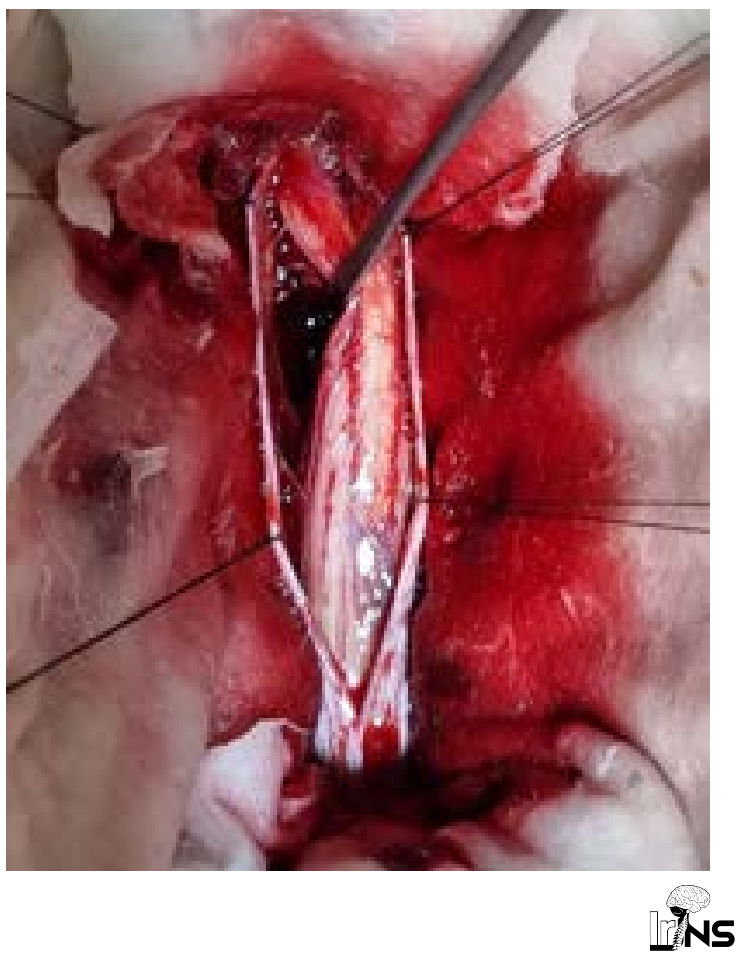

Figure 3. Intra-operative picture presenting intradural hematoma compressing the cord from the anterior aspect

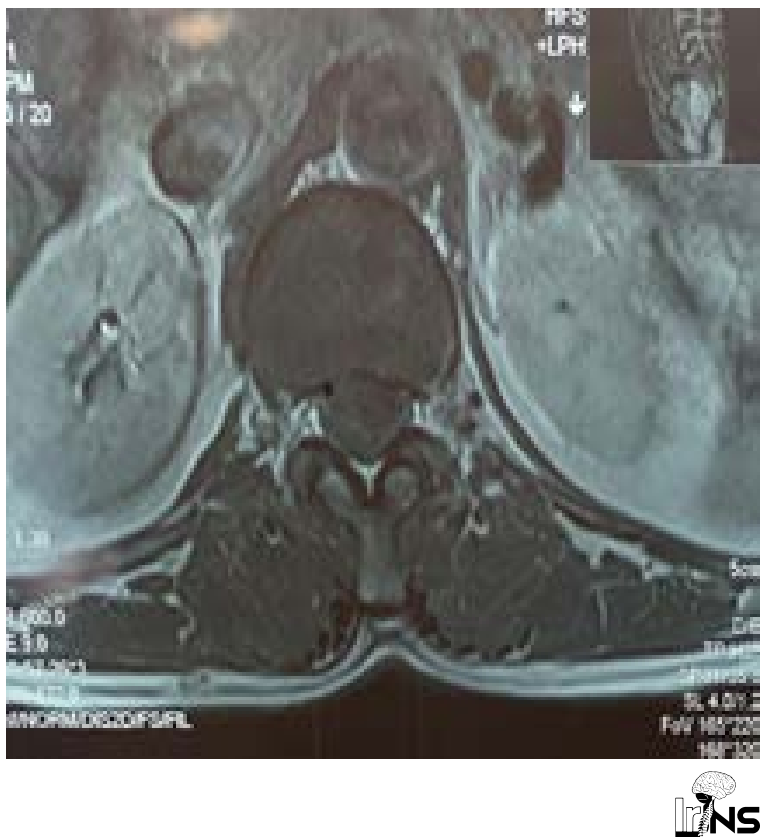

Figure 2. Coronal T2WI of MRI lumbosacral spine illustrating anterior compression of the cord

tion and correlated with the features of organizing hematoma. Post-operatively, the reported patient experienced an uneventful period; however, she continued to be paraplegic at the 6-month follow-up examination.

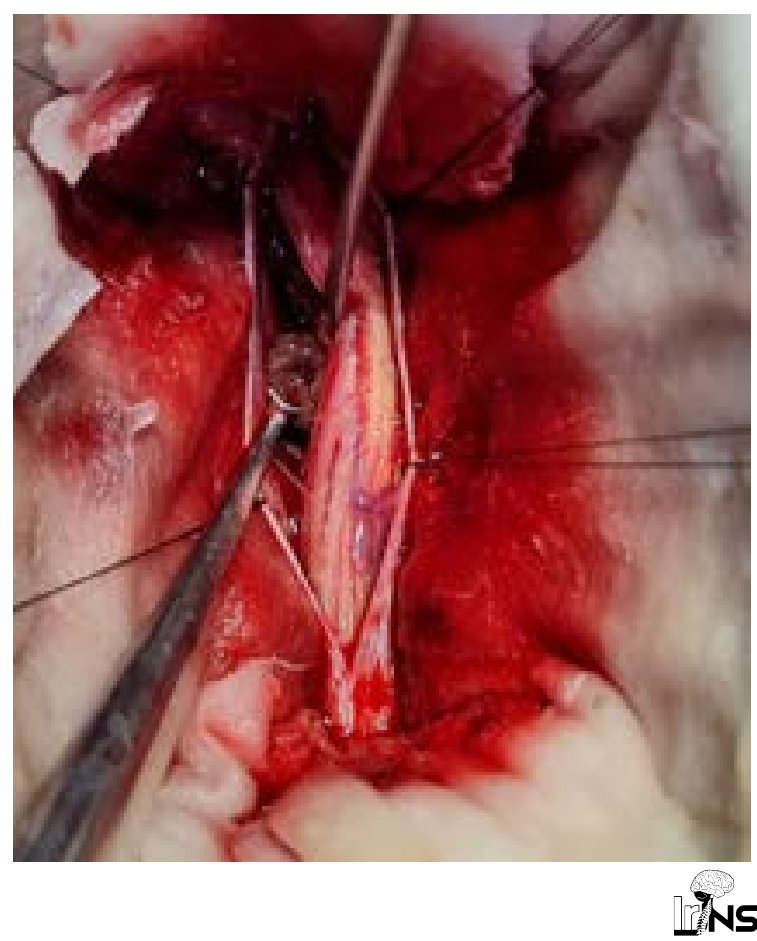

Figure 4. Intra-operative picture manifesting organized intradura hematoma 


\section{Discussion}

latrogenic subdural hematoma is a condition with very low incidence in the general population. Isolated SSH can present symptoms, ranging from straightforward back or neck pain to paraplegia or quadriplegia, depending on the region and size of spinal cord compression [5]. It may be created following lumbar puncture at the needling site or a site slightly distal notwithstanding anyplace in the epidural, subdural, or subarachnoid spaces [6]. A traumatic tap bringing about blood-stained CSF is frequently because of the puncture of the dorsal or ventral epidural venous plexuses and generally manifests no complication. However, any clot which organizes to a significantly larger size compresses the spinal cord or nerve roots; accordingly, this condition leads to significant emergent disability, such as paraplegia and cauda equina syndrome.

Coagulopathy, such as thrombocytopenia, the pre-existing use of anticoagulants, vitamin $\mathrm{K}$ antagonists, antiplatelet medications, and severe prematurity are also attributed to the condition. The nature of the underlying disease which requires the patient to be on long-term anticoagulation may contribute to the poor outcome through a multitude of factors rather than simple hypocoagulability [3]. Transient paraparesis has been reported after post-dural puncture spinal hematoma in a patient receiving ketorolac and as a complication of epidural blood patch [7].

SSH can be difficult to diagnose, preceding the presence of neurological deficits. Thus, the differential diagnosis of SSH should include pulmonary embolism, unconstrained pneumothorax, and acute myocardial infarction without neurological deficit, transverse myelitis, Guillain-Barré Syndrome (GBS), epidural subarachnoid bleeding, and acute spinal cord ischemia with the presence of neurological deficits [8].

There exists literature on early spinal epidural hematoma, clinically mimicking prolapsed disc; however, subdural hematoma mimicking disc prolapse has not been overlooked [9]. In our case, the patient was approached with a diagnosis of Prolapsed Intervertebral Disc (PIVD) presenting with cauda equina syndrome; however, intra-operatively, the findings were suggestive of subdural hematoma.

MRI provides the most valuable visualization of the location and hematoma mass as well as the presence of spinal cord compression. However, there can be occasions where the subdural hematoma might illustrate pictures of prolapsed disc compressing the thecal sac and causing canal stenosis.

Urgent spinal decompression laminectomy and hematoma evacuation have always been the treatment of choice for SSH with severe neurological deficits. However, in few cases, presenting with mild neurological deficits and small hematoma on MRI, spontaneous resolution on dynamic MRI was detected, indicating not requiring surgical intervention and the need for close follow-up on admission. In the current case, we did emergency laminectomy respecting prolapsed disc with CES and intraoperatively found intradural pathology for which a durotomy and hematoma evacuation was performed.

Two salient factors have been suggested to present major prognostic implications, as follows: the degree of preoperative neural deficit and the time interval between ictus and surgery [10]. Patients with minimal symptoms before therapy are more prone to acquire complete recovery than those with major deficits in sensorimotor functioning. There is no guideline framed for the most favorable time for surgery due to the lack of evidence and small sample size of previous studies. The current case was operated on $48 \mathrm{~h}$ after she developed paraplegia due to time taken for further investigations. She continued to be paraplegic with the loss of bowel and bladder control implying the significance of early precise diagnosis and treatment.

\section{Conclusion}

SSH is a rare condition with undiscovered incidence and presents with a spectrum of signs and symptoms. Although MRI is the investigation of choice, SSH presenting, like a prolapsed intervertebral disc on MRI is among its kinds and should be considered. This is because it carries a grave prognosis if not treated early with emergency decompression.

\section{Ethical Considerations}

\section{Compliance with ethical guidelines}

Thorough written informed consent was taken from the patient and her relatives.

Funding

This research did not receive any grant from funding agencies in the public, commercial, or non-profit sectors. 


\section{Authors' contributions}

Data collection: Vishal Singh; Data analysis and interpretation: Vishal Singh; Drafting: Vishal Singh; Critically revising, reviewing submitted version of manuscript: Both authors; Approving the final version of the manuscript: Sajad Arif.

\section{Conflict of interest}

The authors declared no conflict of interest.

\section{References}

[1] Yang NR, Kim SJ, Cho YJ. Cho DS. Spontaneous resolution of nontraumatic acute spinal subdural hematoma. Journal of Korean Neurosurgical Society. 2011; 50(3):268-70. [DOI:10.3340/jkns.2011.50.3.268] [PMID] [PMCID]

[2] Brown MW, Yilmaz TS, Kasper EM. Iatrogenic spinal hematoma as a complication of lumbar puncture: What is the risk and best management plan? Surgical Neurology International. 2016; 7(Suppl 22):S581-9. [PMID] [PMCID]

[3] Gerancher JC, Waterer R, Middleton J. Transient paraparesis after postdural puncture spinal hematoma in a patient receiving ketorolac. Anesthesiology. 1997; 86(2):490-4. [DOI:10.1097/00000542-199702000-00025] [PMID]

[4] Pereira BJA, de Almeida AN, Muio VMF, de Oliveira JG, de Holanda CVM, Fonseca NC. Predictors of outcome in nontraumatic spontaneous acute spinal subdural hematoma: Case report and literature review. World Neurosurgery. 2016; 89:574-7.e7. [DOI:10.1016/j.wneu.2015.11.010] [PMID]

[5] Gopalkrishnan CV, Dhakoji A, Nair S. Spontaneous cervical epidural hematoma of idiopathic etiology: Case report and review of literature. The Journal of Spinal Cord Medicine. 2012. 35(2):113-7. [DOI:10.1179/2045772312Y.0000000001] [PMID] [PMCID]

[6] Goyal A, Dua R, Singh D, Kumar S. Spinal subarachnoid haematoma following lumbar puncture. Neurology India. 1999; 47(4):339-40. https://www.neurologyindia.com/article.asp?issn $=0028-3886$; year $=1999$; volume $=47$;issue $=4$; spage $=339$; epage $=40$; aulast $=$ Goyal

[7] Pai SB, Krishna KN, Chandrashekar S. Post lumbar puncture spinal subarachnoid hematoma causing paraplegia : A short report. Neurology India. 2002; 50(3):367-9. [PMID]

[8] Wang P, Xin XT, Lan H, Chen C, Liu B. Spontaneous cervical epidural hematoma during pregnancy: Case report and literature review. European Spine Journal. 2011; 20(Suppl 2):S176-9. [DOI:10.1007/s00586-010-1484-9] [PMID] [PMCID]

[9] Matsumura A, Namikawa T, Hashimoto R, Okamoto T, Yanagida I, Hoshi M, et al. Clinical management for spontaneous spinal epidural hematoma: Diagnosis and treatment. The Spine Journal. 2008; 8(3):534-7. [DOI:10.1016/j. spinee.2007.01.009] [PMID]

[10] Liao CC, Hsieh PC, Lin TK, Lin CL, Lo YL, Lee SC. Surgical treatment of spontaneous spinal epidural hematoma: A 5-year experience. Journal of Neurosurgery. Spine. 2009; 11(4):480-6. [DOI:10.3171/2009.4.SPINE08904] [PMID] 